\title{
Metastasis to spermatic cord from carcinoma prostate: A case series
}

\author{
Sumit Gahlawat ${ }^{1}$, Sumit Kabra ${ }^{2}$, Arvind Ahuja ${ }^{3}$, Rajeev Sood ${ }^{4}$, Umesh Sharma ${ }^{\mathbf{5}}$, Hemant Kumar Goel \\ From ${ }^{1}$ Assistant Professor, ${ }^{2}$ Senior Resident, ${ }^{4}$ Professor, ${ }^{5}$ Associate Professor, Department of Urology and Renal Transplant, Atal Bihari Vajpayee \\ Institute of Medical Sciences and Dr. Ram Manohar Lohia Hospital, New Delhi, India, ${ }^{3}$ Professor, Department of Pathology, Atal Bihari Vajpayee \\ Institute of Medical Sciences and Dr. Ram Manohar Lohia Hospital, New Delhi, India
}

\begin{abstract}
Despite the high incidence of secondaries to lymph nodes, bones, and lungs in carcinoma (CA) prostate, metastatic involvement of scrotal organs is rare and usually associated with a poor prognosis. Here, we report a case series of three cases of CA prostate with metastatic involvement of scrotal organs. All three patients had metastatic involvement of the spermatic cord, with involvement of epididymis in the first patient and testes in the third patient, revealed incidentally on orchiectomy. Two patients were also found to have coexisting lymphatic filariasis. To date as per the best of our knowledge, only one such case of CA prostate with metastasis to scrotal organs and associated filariasis has been reported. This highlights the need for histopathological evaluation of all orchiectomy specimens. Chronic infection and inflammation leading to lymphatic obstruction due to filariasis probably led to the unusual retrograde spread of the tumor.
\end{abstract}

Key words: Carcinoma prostate, Filariasis, Metastasis, Spermatic cord

$\mathrm{P}$ rostate carcinoma $(\mathrm{CA})$ is one of the most commonly diagnosed malignancies in men. While metastasis to lymph nodes, bones, lungs, and liver are common, there are rare cases of metastasis to the testis, spermatic cord, and epididymis, usually with a poor prognosis. In an autopsy study conducted by Bubendorf et al. among 1589 patients with CA prostate, testicular metastasis was found in only $0.5 \%$, with none of the cases having metastasis to epididymis or spermatic cord [1]. On extensive Pubmed search, we found epididymal metastasis from CA prostate to be reported in less than 30 cases, while metastasis to the cord was even rarer. Our case series highlights the need for histopathological evaluation of all orchiectomy specimens as metastatic involvement of the scrotal organs is considered a sign of advanced disease.

\section{CASE SERIES}

\section{Case 1}

A 61-year-old male presented with lower urinary tract symptoms (both storage and voiding) and low backache for 3 months. The patient was catheterized in view of acute urinary retention 1 month back. On general physical examination, the patient had

\section{Access this article online}

Received - 25 September 2021

Initial Review - 01 October 2021

Accepted - 07-October 2021

DOI: $10.32677 /$ ijcr.v7i10.3093 stable vitals. On local examination, external genitalia was normal. On digital rectal examination (DRE), the prostate was hard and nodular, overlying mucosa was free. His Prostate-Specific Antigen (PSA) was $160 \mathrm{ng} / \mathrm{ml}$. Transrectal ultrasound (TRUS) guided 12 core needle biopsy reported as adenocarcinoma with a modified Gleason score of $8(3+5)$ (Gleason Grade Group 4). On further evaluation, a Technetium-99m bone scan revealed multiple osteoblastic skeletal metastases. Evaluation with contrast-enhanced computed tomography (CECT) of the abdomen and pelvis showed an enlarged prostate with lobulated outlines and heterogeneous enhancement with no lymph node or visceral metastasis. Androgen deprivation therapy (ADT) in the form of surgical castration was performed.

On histopathological examination, grossly both testes showed normal testicular parenchyma. Sections from the left cord revealed tumor deposits (Fig. 1a and b) and sections from the left epididymis showed multiple nests and sheets of tumor deposits, composed of fused glands (Fig. 1c). Tumor cells had hyperchromatic nuclei, a high $\mathrm{N}$ : C ratio, inconspicuous nucleoli, and scant to moderate cytoplasm. In addition, many transverse sections of adult filarial worms were identified in the lymphatic channels of the spermatic cord on microscopic examination (Fig. 1d). Immunohistochemistry staining for PSA was weakly positive (Fig. 2a), however, a-methyl acyl-coenzyme A racemase (AMACR) (Fig. 2b) and Pancytokeratin were positive. Based

Correspondence to: Dr. Sumit Kabra, Department of Urology and Renal Transplant, Atal Bihari Vajpayee Institute of Medical Sciences and Dr. Ram Manohar Lohia Hospital, New Delhi, India. E-mail: sumitdrkabra1988@gmail.com

(C) 2021 Creative Commons Attribution-NonCommercial 4.0 International License (CC BY-NC-ND 4.0). 


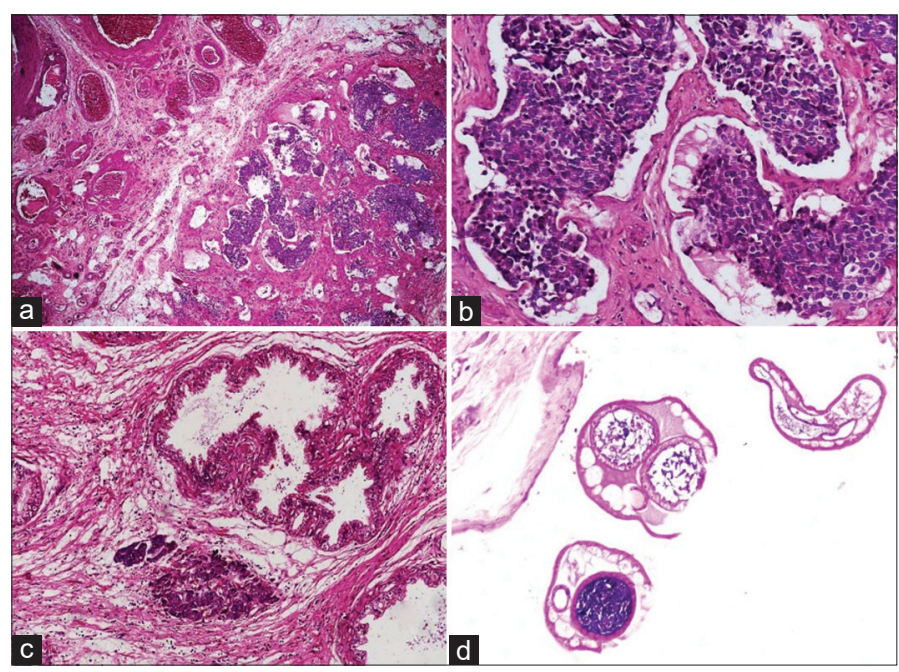

Figure 1: Histopathology (a) Low power photomicrograph showing emboli in the lymphatics of spermatic cord (Hematoxylin and Eosin [H and E], 10×), (b) Medium power showing multiple nests and sheets of tumour deposits composed of fused glands (H and E, 20×), (c) Tumour deposits in the epididymis $(\mathrm{H}$ and $\mathrm{E}, 20 \times)$, (d) Transverse sections of adult filarial worm (H and $\mathrm{E}, 20 \times$ ) (Case 1)

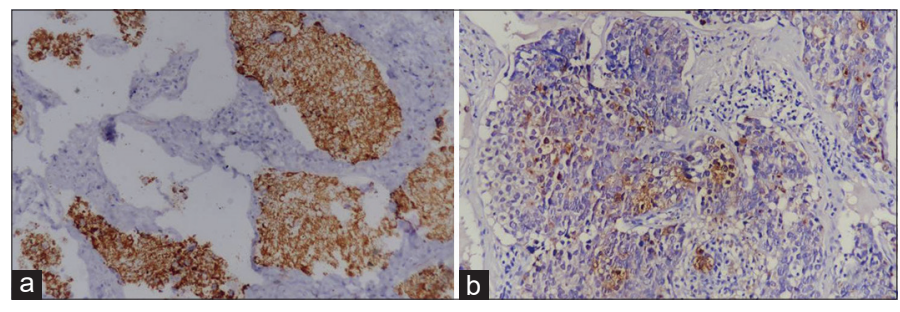

Figure 2: (a) Immunohistochemistry (IHC)-a-methyl acyl-coenzyme A racemase showing diffuse positivity, (b) IHC-Prostate-Specific Antigen showing weak positivity (Case 1)

on histopathological and immunohistochemical findings, the diagnosis was compatible with metastasis from CA prostate. The patient has been on follow-up for the past 6 months with the stable disease according to RECIST 1.1 criteria and a serum PSA level of $1 \mathrm{ng} / \mathrm{ml}$.

\section{Case 2}

A 58-year-old male presented with lower urinary tract symptoms (both storage and voiding) for 4 months. On general physical examination, the patient had stable vitals. On local examination, external genitalia was normal. DRE revealed a hard and nodular prostate. His PSA was $210 \mathrm{ng} / \mathrm{ml}$. TRUS-guided 12 core needle biopsy was reported as adenocarcinoma with a modified Gleason score of $10(5+5)$ (Gleason Grade Group 5). Technetium-99m bone scan revealed multiple osteoblastic skeletal metastases. CECT of the abdomen and pelvis showed an enlarged prostate showing heterogeneous contrast enhancement with few calcifications within and multiple lymph nodes along bilateral internal iliac vessels and para-aortic region. ADT in the form of surgical castration was performed.

On histopathological examination, both testes showed normal testicular parenchyma. Section from the spermatic cord revealed

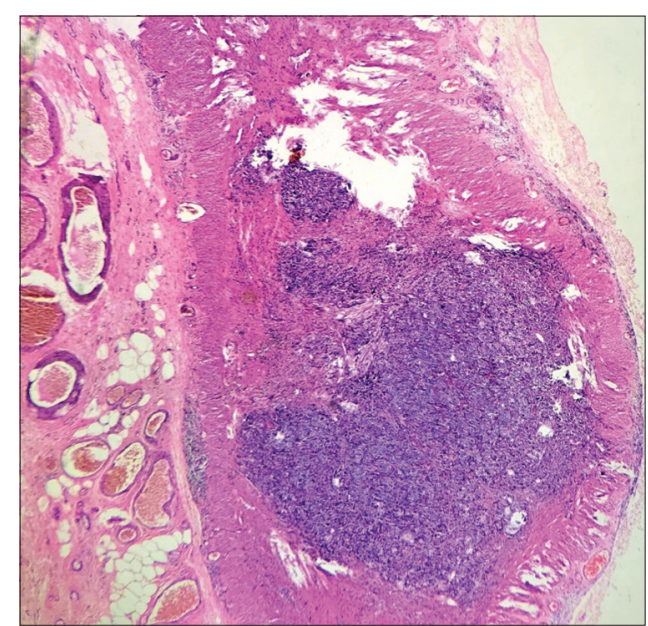

Figure 3: Photomicrograph showing vascular emboli in the spermatic cord (Case 2)

tumor deposits (Fig. 3). The patient has been on follow-up for the past 4 months and has completed six cycles of Injection Docetaxel $120 \mathrm{mg}$ with a current PSA level of $20 \mathrm{ng} / \mathrm{ml}$ and has progressive disease according to RECIST 1.1 criteria.

\section{Case 3}

A 61-year-old male, known hypertensive, with a history of chronic kidney disease, presented with total painless gross hematuria and lower urinary tract symptoms (both storage and voiding) for 3 months. On general physical examination, pulse was $88 / \mathrm{min}$ and blood pressure was $130 / 80 \mathrm{~mm}$ of $\mathrm{Hg}$. On local examination, external genitalia was normal and DRE revealed a hard and nodular prostate. Urine for malignant cytology was negative for malignant cells, however, revealed very occasional sheathed microfilaria. His PSA was $80 \mathrm{ng} / \mathrm{ml}$. TRUS-guided 12 core needle biopsy reported as adenocarcinoma with a modified Gleason score of 9 (5+4) (Gleason Grade Group 5). On metastatic workup, Technetium-99m bone scan revealed multiple osteoblastic skeletal metastases. Evaluation with non-contrast computed tomography of the abdomen and pelvis revealed a large soft tissue mass-like lesion $(71 \times 48 \mathrm{~mm})$ in the region of the base of urinary bladder indistinguishable from prostate with enlarged retroperitoneal and pelvic lymph nodes. The patient underwent bilateral simple orchiectomy as part of ADT.

On histopathological examination, grossly testicular parenchyma appeared unremarkable. Section from both testes and left spermatic cord showed tumor deposits (Fig. 4a and b). Sections from epididymis were free of metastatic deposits. The patient was started on Injection Docetaxel $120 \mathrm{mg}$ but expired after 2 months of diagnosis.

\section{DISCUSSION}

Simultaneous involvement of spermatic cord and epididymis is very rare, with only two cases being reported in the literature. The most probable mechanisms of dissemination are retrograde venous extension, retrograde lymphatic extension, and arterial 


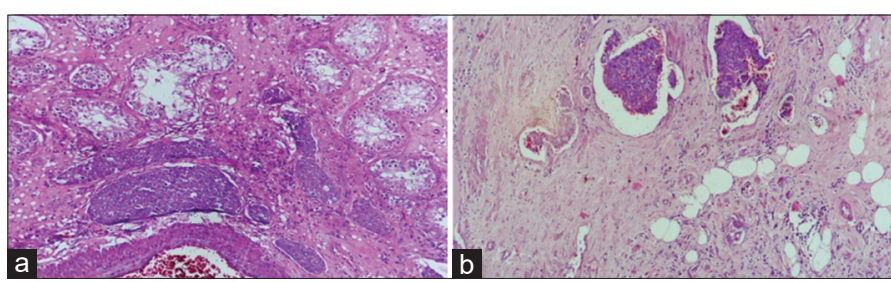

Figure 4: (a) Section from testes showing metastatic nests of carcinoma between the seminiferous tubules (Hematoxylin and Eosin [H and E], 200 $\times$ ), (b) Spermatic cord showing lymphovascular tumour emboli (H and E, 200×) (Case 3)

Table 1: Review of the Literature: Spermatic cord metastasis from carcinoma prostate

\begin{tabular}{llcll}
\hline Case No. & Author (Year) & Age & Side & $\begin{array}{l}\text { Involved } \\
\text { structure }\end{array}$ \\
\hline 1 & $\begin{array}{l}\text { Thompson and Pilcher } \\
\text { (1905) [6] }\end{array}$ & 69 & $\begin{array}{l}\text { Left } \\
\text { side }\end{array}$ & $\begin{array}{l}\text { Spermatic cord } \\
\text { and epididymis }\end{array}$ \\
2 & Young (1935) [7] & 65 & $?$ & Spermatic cord \\
3 & Young (1935) [7] & 64 & $?$ & Spermatic cord \\
4 & Wilenius and Seppänen & - & - & Spermatic cord \\
& (1969) [8] & & & \\
5 & Johansson and Lannes & 84 & Right & Spermatic cord \\
& (1983) [9] & & & \\
6 & $\begin{array}{l}\text { Johansson and Lannes } \\
\text { (1983) [9] }\end{array}$ & 67 & B/L & $\begin{array}{l}\text { Spermatic cord } \\
\text { and epididymis }\end{array}$ \\
7 & $\begin{array}{l}\text { Johansson and Lannes } \\
\text { (1983) [9] }\end{array}$ & 79 & Left & Spermatic cord \\
8 & Bawa et al. (2003) [10] & 85 & Left & Spermatic cord \\
9 & Gergelis et al. (2019) [11] & 65 & Left & Spermatic cord \\
\hline
\end{tabular}

embolism [2]. Hennigar and Hooper [3] hypothesized retrograde extension through the vas deferens as a possible mechanism of spread. Periductal lymphatic vessels have been demonstrated around the vas deferens [4]. The major lymphatic trunks have valves that ensure lymphatic flow in a unidirectional centripetal fashion. However, in any condition leading to obstruction of lymphatics, the vessels dilate leading to the incompetence of the valves allowing bidirectional flow. This provides a mechanism for the retrograde metastasis of malignant tumors. Two of four patients had quiescent filarial infestation which may have led to the blockade of the lymphatic vessels. The presence of adult filarial worms can trigger a series of inflammatory events causing disruption of lymphatic integrity and function. These inflammatory mediators act on the lymphatic endothelial cells and result in obliterative lymphangitis causing dilatation, hypertrophy, and varicosity of the lymphatics with peripheral lymph stasis [5]. Filarial infection in our patients may possibly have led to the retrograde extension of the tumor due to lymphatic blockade.

In the first case, immunohistochemical staining was weakly positive for PSA but AMACR positive, making it unique. PSA stain, a sensitive and specific biomarker of prostatic differentiation may show only a weak positivity in poorly differentiated tumors. In such diagnostic dilemmas, AMACR, the prostate biomarker with very high sensitivity and specificity can be used as an adjuvant.

In the literature review, we could find only two cases having metastasis to the epididymis and spermatic cord and seven cases having isolated spermatic cord metastasis from prostatic adenocarcinoma (Table 1) [6-11].

To the best of our knowledge, this is the first case series of CA prostate with metastasis to scrotal organs and associated filariasis. Our series highlights the need for histopathological evaluation of all orchiectomy specimens. Metastatic involvement of the scrotal organs is considered a sign of advanced disease with unfavorable prognosis.

\section{CONCLUSION}

CA prostate with metastasis to spermatic cord and epididymis is very rare. Chronic infection and inflammation leading to lymphatic obstruction due to filariasis probably led to this unusual retrograde spread of tumor.

\section{REFERENCES}

1. Bubendorf L, Schöpfer A, Wagner U, Sauter G, Moch H, Willi N, et al. Metastatic patterns of prostate cancer: An autopsy study of 1, 589 patients. Hum Pathol 2000;31:578-83.

2. Pienkos EJ, Jablokow VR. Secondary testicular tumors. Cancer 1972;30:481-5.

3. Hennigar KS, Hooper JW Jr. Carcinoma of the testis metastatic from carcinoma of the prostate. AMA Arch Pathol 1954;57:121-9.

4. Bruhns C. Untersuchungen über die lymphgefässe und lymphdrüsen der prostata des menschen. Arch F Anat U Physiol 1904;330-49.

5. Bennuru S, Nutman TB. Lymphatics in human lymphatic filariasis: In vitro models of parasite-induced lymphatic remodeling. Lymphat Res Biol 2009; 7:215-9.

6. Thompson GJ, Pilcher F. Carcinoma of the vas deferens: Report of a case. J Urol 1935;34:714-7.

7. Young HH. The early diagnosis and radical cure of carcinoma of the prostate: Being a study of 40 cases and presentation of a radical operation which was carried out in four cases. Johns Hopkins Hosp Bull 1905;16:315-21.

8. Wilenius R, Seppänen J. Two unusual cases of metastasis of prostatic cancer: Metastases to the spermatic cord and the epididymis. Ann Chir Gynaecol Fenn 1969;58:172-5.

9. Johansson JE, Lannes P. Metastases to the spermatic cord, epididymis and testicles from carcinoma of the prostate-five cases. Scand J Urol Nephrol 1983;17:249-51.

10. Bawa AS, Singh R, Bansal VK, Punia RS. Spermatic cord metastasis from prostatic cancer. J Postgrad Med 2003;49:97-8.

11. Gergelis KR, Remme DS, Choo CR. Isolated biopsy-proven recurrence of prostate carcinoma in the spermatic cord after radical prostatectomy detected with 11C-choline PET/CT. Urol Case Rep 2019;26:100985.

Funding: None; Conflicts of Interest: None Stated.

How to cite this article: Gahlawat S, Kabra S, Ahuja A, Sood R, Sharma U, Goel HK. Metastasis to spermatic cord from carcinoma prostate: A case series. Indian J Case Reports. 2021;7(10):424-426. 